Original article

\title{
Preliminary study of clinical outcomes for single implant-retained mandibular overdentures
}

\author{
Mari Asami $^{1)}$, Manabu Kanazawa ${ }^{1)}$, Thuy V. Lam ${ }^{1)}$, Khaing M. Thu' ${ }^{1)}$, Daisuke Sato ${ }^{2}$, and Shunsuke Minakuchi ${ }^{1)}$ \\ ${ }^{1)}$ Department of Gerodontology and Oral Rehabilitation, Graduate School of Medical and Dental Sciences, Tokyo Medical and Dental University, Tokyo, Japan \\ ${ }^{2}$ Department of Implant Dentistry, School of Dentistry, Showa University, Tokyo, Japan
}

(Received February 21, 2019; Accepted June 24, 2019)

\begin{abstract}
This study evaluated marginal bone loss and prosthetic complications associated with single implant-retained mandibular overdentures (1-IODs) with locator attachments. The 1-IOD was placed in the mandibular midline by using a conventional loading protocol in 22 patients with an edentulous mandible. Marginal bone loss at the start of loading and 12 months postoperatively was assessed by radiographic and crestal bone evaluation. The crestal bone was defined as the distance between the customized abutment shoulder and the top of the bone, as indicated by probing. In addition, implant stability quotient and prosthetic complications were recorded. The cumulative implant survival rate was $95.5 \%$. Median implant stability quotient remained greater than 80 , and median radiographic bone loss was $0.56 \mathrm{~mm}$. Crestal measurement showed a median crestal bone loss of $0.16,0.43,0.39$, and $0.52 \mathrm{~mm}$ on the buccal, right, lingual, and left sides, respectively. Both radiographic and crestal bone loss values significantly differed between the start of implant loading and 12 months postoperatively (except on the buccal and lingual sides; $P<0.05$ ). The need to replace the nylon insert was the most common complication. Conventional loading of a 1-IOD with a locator attachment resulted in a high survival rate, good implant stability, and acceptable marginal bone loss.
\end{abstract}

Keywords; marginal bone loss, prosthetic complications, single implantretained mandibular overdentures

\section{Introduction}

Placement of 2 implant-supported overdentures (2-IODs) is a currently accepted treatment for edentulous individuals $[1,2]$. However, as the number of community-dwelling elderly adults increases, treatment plans that entail the least risk are required [3]. One such low-risk treatment option is a single implant-retained overdenture (1-IOD) in the middle of the mandible - a low-cost procedure that results in less risk and patient satisfaction similar to that reported for conventional 2-IODs [4].

Locator attachments are increasingly used with implant overdentures. The advantages of a locator attachment are that an appropriate retention force can be chosen from several types of nylon available and that little time is required for replacement of the nylon. Furthermore, use of a locator attachment is cost-effective [5-8] and allows implants to be placed at varying inclinations. When an implant is placed, especially in the anterior mandible, the implant tends to be buccally or lingually inclined because of the anatomy of the ridge in this area. However, use of an appropriate insert prevented the decrease in retention in patients with angulated implants $[9,10]$. Locator attachments are now frequently used with 1-IODs [11-13]. Implant survival rate, implant stability, marginal bone loss, and complications must be considered when evaluating the suitability of a locator attachment for a 1-IOD. Implant survival rates were reported to be $100 \%$ for conventional and immediate loading on a locator-retained 1-IOD

Correspondence to Dr. Manabu Kanazawa, Department of Gerodontology and Oral Rehabilitation, Graduate School of Medical and Dental Sciences, Tokyo Medical and Dental University, 1-5-45 Yushima, Bunkyo-ku, Tokyo 113-8510, Japan

Fax: +81-3-5803-5586 E-mail: m.kanazawa.gerd@tmd.ac.jp

Color figures can be viewed in the online issue at J-STAGE.

doi.org/10.2334/josnusd.19-0079

DN/JST.JSTAGE/josnusd/19-0079
$[11,13]$. Implant stability can be measured by resonance frequency analysis and expressed as the implant stability quotient (ISQ). In a previous study, the ISQ increased from 73.6 to 75.1 on immediate loading of a 1-IOD with a locator [11]; however, no studies have evaluated conventional loading. Furthermore, marginal bone loss is an essential direct biological indicator of IOD prognosis. Previous studies reported marginal bone loss of $1.5 \mathrm{~mm}$ at 12 months after conventional loading with a ball attachment [14]; 0.6 $\mathrm{mm}$ (right side) and $0.7 \mathrm{~mm}$ (left side) [15], $0.4 \mathrm{~mm}$ [16], and $0.2 \mathrm{~mm}$ [11] after immediate loading; and $0.6 \mathrm{~mm}$ [17] after early loading at 12 months. However, with regard to locator attachment, only immediate loading has been studied, and a mean marginal bone loss of $0.23 \mathrm{~mm}$ was observed [11]. The criterion for success is considered to be less than $1.0 \mathrm{~mm}$ of bone loss during the first year of function and less than $0.2 \mathrm{~mm}$ annually thereafter $[18,19]$. In general, radiographic imaging is used to evaluate marginal bone loss on the right and left sides; however, marginal bone loss on the buccal and lingual sides has not been evaluated in patients with a 1-IOD. Crestal bone measurements were recently reported to be effective in evaluating bone height around the implant (buccal, lingual, right, left) when a $2-I O D$ was used $[20,21]$ and may also be applicable when a 1-IOD is used.

With respect to the prosthetics, locator attachments require periodic nylon replacement. Furthermore, previous studies of 2-IODs suggest that locator attachment has disadvantages, such as accumulation of food and plaque at the site of the locator abutment and difficulty wearing the denture $[6,22]$. Therefore, an overview of the 1-IOD from the perspective of prosthetics is required.

Few studies have evaluated 1-IODs that use a locator attachment, particularly in relation to the stability of such implants, the amount of marginal bone loss, and other complications under conventional loading conditions. This study assessed survival rate, stability, amount of marginal bone loss (as estimated by radiographic and crestal bone measurements), and other complications of 1-IODs with a locator attachment.

\section{Materials and Methods}

\section{Study design}

Twenty-two patients (10 men, 12 women; age range 61-91 years) with an edentulous mandible were recruited at the Dental Hospital of Tokyo Medical and Dental University from October 2015 through March 2016. The study inclusion criteria were presence of an edentulous mandible for at least 6 months; sufficient bone height ( $\geq 13 \mathrm{~mm})$ and width $(\geq 6.1 \mathrm{~mm})$ at the mandible midline; written and spoken Japanese skills adequate to respond to questionnaires; and age older than 50 years. Patients were excluded if they had a medical contraindication to implant surgery, were receiving bisphosphonate therapy at the time of the study, had a history of chemotherapy or radiation therapy to the head or neck, smoked $>20$ cigarettes a day, or had an infection, a psychiatric disorder, a temporomandibular disorder, dry mouth, or oral dyskinesia.

The sample size required for this study was estimated by using $100-\mathrm{mm}$ Visual Analog Scale (VAS) scores for patient satisfaction, as described in the trial protocol of Kanazawa et al. [23]. Twenty participants were required for $80 \%$ power with a 2 -sided alpha level of 0.05 , when VAS scores were assumed to be normally distributed. Twenty-two participants were recruited, to allow for dropouts. The study protocol was approved by the Ethics Committee of the Faculty of Dentistry, Tokyo Medical and Dental 


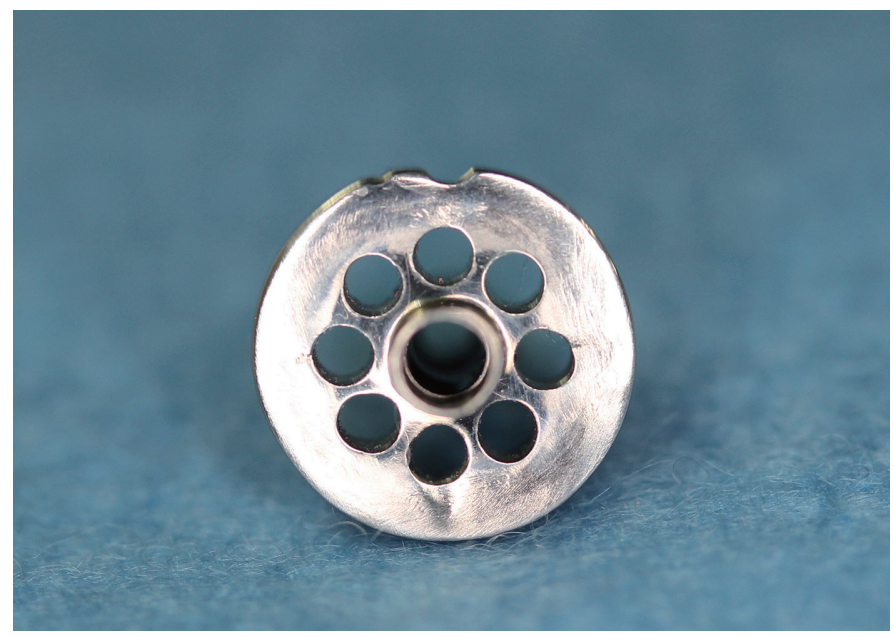

Fig. 1 Superior view of the customized abutment. Four of the 8 holes were selected for measurement as the buccal, right, lingual, and left sides.

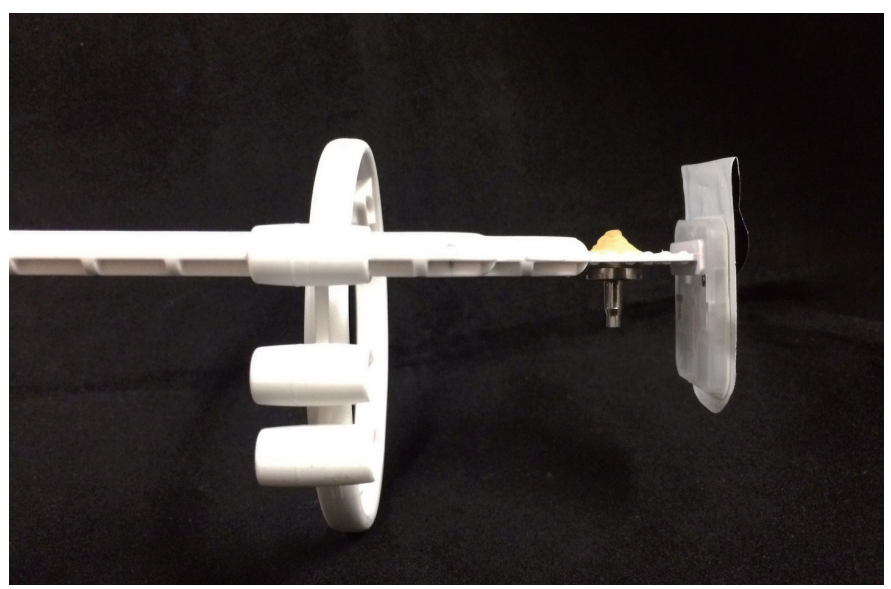

Fig. 3 Lateral view of the customized radiographic guide. The top of the abutment was placed in the hole of the bite wing.

University (registration number 1162) and has been published in the UMIN Clinical Trials Registry (UMIN-CTR, trial number UMIN000017883). All experiments were conducted in accordance with the Declaration of Helsinki (http://www.wma.net). Informed consent was obtained from all participants before their entry into the trial.

\section{Surgical procedures}

All participants received an appropriate new mandibular complete denture before placement of the implant. A crestal incision was made to elevate a full-thickness flap. A single implant (SLA BLT $4.1 \times 10 \mathrm{~mm}$, Straumann AG, Basel, Switzerland) was placed in the mandibular midline by following the recommended surgical protocol. Once placed, the implant was covered by a healing cap. Maximum insertion torque was measured with a manual torque wrench. The mucosal surface of the complete denture around the implant was relieved. Three to 5 months later, a locator abutment (Locator, Zest Anchors, Escondido, CA, USA) was connected to the implant, and a locator cap was placed on the mucosal surface of the complete denture.

\section{Outcomes}

Implant stability at the time of implant placement $\left(\mathrm{T}_{0}\right)$, at the time loading was started $\left(\mathrm{T}_{\mathrm{L}}\right)$, and at the 12-month postoperative visit $\left(\mathrm{T}_{12}\right)$ was recorded by using a resonance frequency analysis device (Osstell, Osstell AB, Gothenburg, Sweden) with a smart peg attached to the implant. Implant stability was measured from the buccolingual and mesiodistal directions, and mean values were used in the data analysis.

Marginal bone loss was measured indirectly by radiographic measurement and directly by measurement of the crestal bone by probing. The

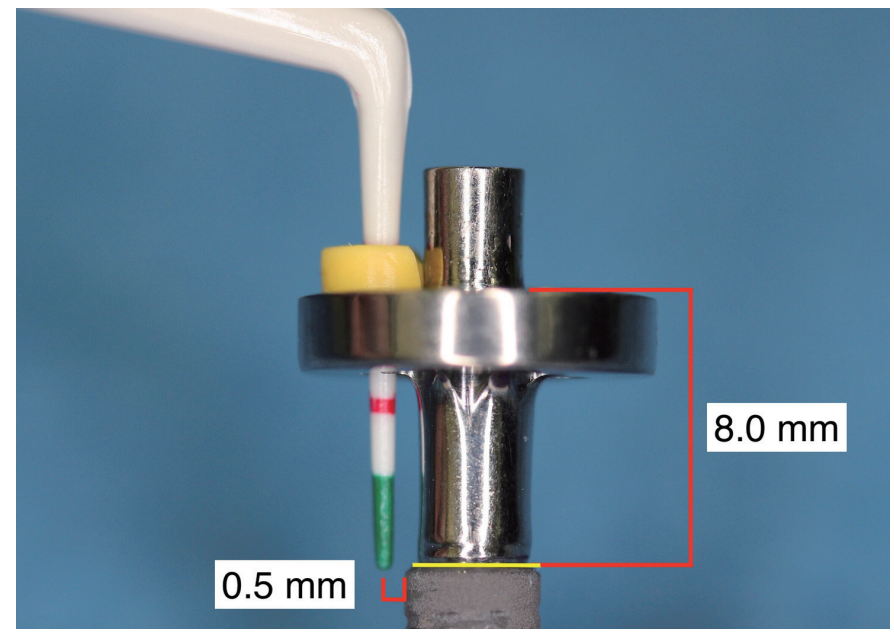

Fig. 2 Lateral view of the customized abutment. The height of the abutment is $8.0 \mathrm{~mm}$. The probing position was reproducible because of an internal groove in the implant. The abutment holes were designed so that they did not touch the implant shoulder. Probing was maintained at a distance of $0.5 \mathrm{~mm}$ from the implant shoulder.

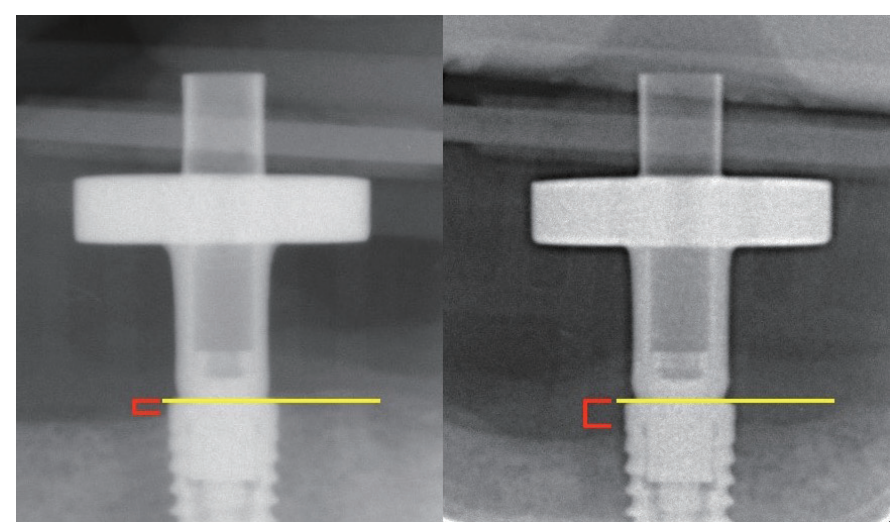

Fig. 4 Radiographic marginal bone loss. The reference point is the top of the shoulder of the implant (yellow line). The distance between the reference point and the most coronal point where the bone touched the implant was measured (red mark). Marginal bone loss at $T_{12}$ was defined as the difference between the values at $\mathrm{T}_{0}$ and $\mathrm{T}_{12}$.

distance from the implant shoulder to the first bone-to-implant contact was evaluated at $\mathrm{T}_{0}, \mathrm{~T}_{\mathrm{L}}$, and $\mathrm{T}_{12}$. Both measurements utilized a modified "BB gauge" method. The BB gauge is a customized machined abutment developed by Brochu et al. to measure crestal bone height [20]. The gauge is equipped with a platform perpendicular to the abutment and contains holes for taking measurements with the probes (Figs. 1 and 2); the probing hole position is reproducible because of an internal groove in the implant. The reference point for both measurements was the top of the implant, which was selected because the distance from the top of the crestal bone to the top of the implant was regarded as the implant placement depth. At $\mathrm{T}_{0}$, the bone level above the reference point was corrected to 0 . A positive value from the reference point corresponded to coronal bone and a negative value to apical bone. Marginal bone loss was calculated as the difference in the values for the 2 time points; the value at $T_{L}$ was regarded as marginal bone loss at baseline.

Standardized periapical radiographs produced by the long-cone technique with a standardized film holder (Uni-Grip, Dentsply Sirona, York, PA, USA) were used for the radiographic measurements, and a customized abutment was used when repeating the measurements (Fig. 3). The top of the abutment was placed in the hole of the bite wing. The distance between the reference point and the first bone-to-implant contact was measured on the right and left sides of the implant with ImageJ software (version $1.51 \mathrm{~s}$, National Institutes of Health, Bethesda, MD, USA; Fig. 4). The interpreted value was calibrated based on the diameter of the implant. The mean value of both sides at each time point was used in the analysis.

A customized abutment was used to measure crestal bone loss on the buccal, lingual, right, and left sides of the implant; 4 of the 8 holes were 


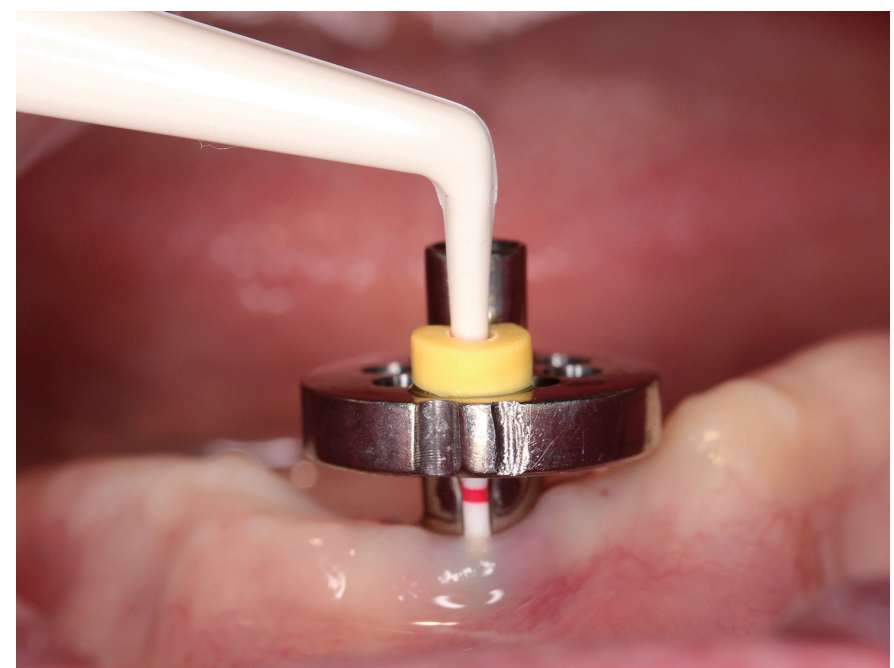

Fig. 5 Crestal bone measurement with the customized abutment

Table 1 Results of radiographic assessment of marginal bone loss at 2 time points

\begin{tabular}{lll}
\hline Start of implant loading & 12 months postoperatively & Wilcoxon signed-rank test \\
\hline$\left(\mathrm{T}_{\mathrm{L}}\right)(n=21)$ & $\left(\mathrm{T}_{12}\right)(n=21)$ & $(P$-value $)$ \\
Median [range] & Median [range] & \\
$0.11[0,0.56]$ & $0.56[0.24,0.85]$ & $<0.01$ \\
\hline $\mathrm{T}_{\mathrm{L}}$, start of implant loading; $\mathrm{T}_{12}, 12$ months postoperatively &
\end{tabular}

Table 2 Results of crestal bone measurement of marginal bone loss at 2 time points

\begin{tabular}{llll}
\hline Probing position & $\mathrm{T}_{\mathrm{L}}(n=21)$ & $\mathrm{T}_{12}(n=21)$ & $\begin{array}{l}\text { Wilcoxon signed-rank test } \\
\text { between } \mathrm{T}_{\mathrm{L}} \text { and } \mathrm{T}_{12}\end{array}$ \\
\cline { 2 - 4 } & Median [range] & Median [range] & $(P$-value $)$ \\
Buccal & $0.07[-0.41,1.01]$ & $0.16[-0.33,0.56]$ & 0.75 \\
Right & $0.12[0,0.64]$ & $0.43[0.15,0.95]$ & 0.01 \\
Lingual & $0.03[0,0.45]$ & $0.39[0,0.78]$ & 0.09 \\
Left & $0.08[0,0.39]$ & $0.52[0.05,0.88]$ & 0.03 \\
\hline $\mathrm{T}_{\text {L }}$ start of implant loading: $\mathrm{T}_{12}, 12$ months postoperatively
\end{tabular}

selected for measurement in each case. After insertion of plastic pocket probes (Perio, KerrHawe SA, Bioggio, Switzerland) with rubber stoppers, a digital scale was used to determine the precise depth. Local anesthetic was applied to the gingiva around the implant as the probe went through the gingiva and touched the bone surface, and the distance was measured between the customized abutment shoulder and the top of the bone (Fig. 5 ). The value at each time point was calculated from the reference point by subtracting the actual measured value from $8.0 \mathrm{~mm}$ (the height of the customized abutment; Fig. 2).

Details of prosthetic maintenance, including denture repair, relining, nylon replacement, and repositioning of the metal housing, were recorded from $\mathrm{T}_{\mathrm{L}}$ to $\mathrm{T}_{12}$.

\section{Statistical analysis}

The cumulative implant survival rate was evaluated by Kaplan-Meier analysis. Implant stability and radiographic and crestal bone loss values were not normally distributed. The differences in implant stability between the different observation time points and between the crestal bone loss measurement sites were analyzed with the Friedman test followed by the Wilcoxon signed-rank test. Differences between $T_{L}$ and $T_{12}$ in radiographic and crestal bone loss were evaluated with the Wilcoxon signed-rank test. All analyses were performed by using SPSS for Windows (version 16.0; SPSS Inc., Chicago, IL, USA). A $P$-value of $<0.05$ was considered to indicate statistical significance.

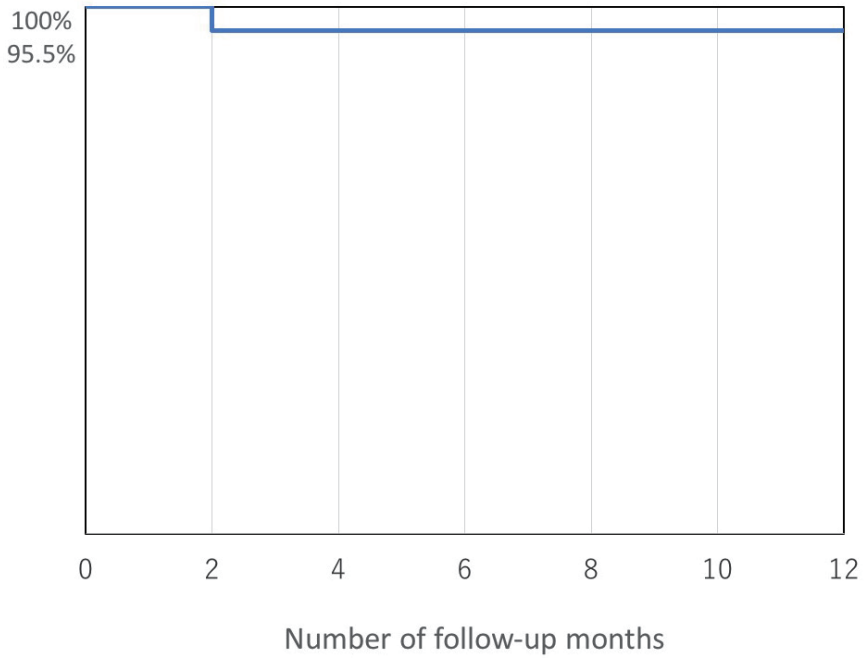

Fig. 6 Cumulative implant survival rates. One participant lost an implant at 2 months postoperatively, thus yielding an implant survival rate of $95.5 \%$.

Table 3 Prosthodontic complications during 12 months of postoperative follow-up

\begin{tabular}{lc}
\hline Prosthodontic event & $n$ \\
\hline Nylon replacement & 32 \\
Repositioning of metal housing & 9 \\
Denture reline & 4 \\
Denture fracture & 2 \\
Denture replacements & 2 \\
All events & 49 \\
\hline
\end{tabular}

\section{Results}

Twenty-two edentulous patients ( 10 men, 12 women; mean age 74.2 [range 61-91] years) were enrolled in the study. Fourteen patients were fully edentulous and 8 patients had some upper teeth. The 22 implants were inserted by the same experienced operator. At 2 months postoperatively, 1 patient had lost the implant and refused a new one; thus, 21 patients were available for follow-up until the 12-month time point (cumulative implant survival rate, 95.5\%; Fig. 6).

\section{Implant stability}

ISQ values greater than 65 were recorded for all 22 implants at the time of placement. The median ISQ for the 21 surviving implants was 82.5 at the time of placement, 82.0 at the start of loading, and 81.0 at the 12-month postoperative visit. There was no significant change in ISQ during the observation period $(P=0.16)$.

\section{Marginal bone loss}

The median radiographic bone loss was $0.56 \mathrm{~mm}$ (Table 1). One of the 21 patients had a bone loss of $>2 \mathrm{~mm}$. The Wilcoxon signed-rank test showed a significant difference in bone loss between $\mathrm{T}_{\mathrm{L}}$ and $\mathrm{T}_{12}(P<0.01)$.

The results for crestal bone loss are shown in Table 2. Median bone loss at $\mathrm{T}_{12}$ was $0.16 \mathrm{~mm}$ on the buccal side, $0.43 \mathrm{~mm}$ on the right side, $0.39 \mathrm{~mm}$ on the lingual side, and $0.52 \mathrm{~mm}$ on the left side. The Wilcoxon signed-rank test showed significant differences between bone loss values at $\mathrm{T}_{\mathrm{L}}$ and $\mathrm{T}_{12}$ on the right and left sides $(P<0.05)$. The Friedman test showed no significant difference in crestal bone loss between these positions at either time point $(P>0.05)$.

\section{Complications}

There were 49 recorded events for the 21 overdentures during the period from $T_{L}$ to $T_{12}$. The most common complication was need for replacement of nylon insert because of loss of retention or deformation of the nylon insert (Table 3). Half these nylon replacements were required during the first 2 months after setting the locator. Two of the 21 patients required 3 replacements, and 1 required 4 replacements because of early loss of reten- 
tion due to nylon deformation in the first 2 months. Nine patients needed repositioning of the metal housing, and 4 patients needed relines in order to improve stability. Two fractures occurred at the denture midline in 1 patient, who then required a new denture. New dentures were also required for a patient who experienced a $15-\mathrm{mm}$ reduction in bone.

\section{Discussion}

In this preliminary study, conventionally loaded 1-IODs with a locator attachment had a high survival rate, good stability, and acceptable marginal bone loss, as indicated by both types of evaluation. Previous studies using conventional loading reported survival rates of $100 \%[13,24]$ and $98.5 \%$ [25]; however, in this study, one implant was lost during the healing period; thus, the survival rate was $95.5 \%$. Analysis of implant stability showed a slight decrease in ISQ, although median ISQ remained greater than 80 during the study period. This is consistent with the findings of previous studies, which reported that initial stability was high and decreased only slightly thereafter [26,27]. It should be noted that the present implant had a tapered design and a deep thread; thus, primary stability was more easily achieved than in earlier studies.

Median marginal bone loss on conventional radiographic measurements was within the previously reported range [14-17] and consistent with criteria for success $[18,19]$. Marginal bone loss was large $(2 \mathrm{~mm})$ in a patient with connective tissue disease. The substantial marginal bone loss in patients with connective tissue disease has been attributed to diminished mucosal vascularization during disease pathogenesis [28,29].

The crestal bone measurement results showed a significant difference in marginal bone loss between $T_{L}$ and $T_{12}$, except for the buccal and lingual sides. Misch reported that early bone loss usually occurred on the buccal side of implants [30]. Liu et al. used a finite element model to evaluate movement of the denture base and strain on the peri-implant cortical bone when a 1-IOD was placed and found that inclined loading on the first molar resulted in greater strain on the loaded and lingual sides of the bone than at the other positions [31]. However, they also reported that maximum stress was below the physiological tolerance threshold of the bone. In the present study, buccal and lingual bone loss at 1 year was acceptable when compared with that on the other sides, which is consistent with the findings of Liu et al. [31]. Bone loss may be slower on the buccal and lingual sides than on the right and left sides; however, a longer observation period is required in order to confirm this.

Implant diameter and angle of insertion should also be considered. An in vitro study indicated that buccolingual bone loss increases with increasing implant diameter [32]. Therefore, buccolingual marginal bone loss might be greater if an implant with a small diameter is placed in a narrow bone ridge. Another report suggested that buccal inclination of an implant increased peri-implant stresses [33]. The clinical effect of buccal inclination of an implant with a 1-IOD has not been evaluated. Further investigation of the association between implant inclination and marginal bone loss is necessary. In this study, marginal bone loss was evaluated with probing rather than with computed tomography (CT) images because artifacts could complicate assessment of the amount of bone loss. However, probing is invasive and can damage attachment between the implant surface and connective tissue. Using cone-beam CT, which results in greater radiation exposure, Elsyad et al. evaluated buccolingual marginal bone loss in 2-IODs and found that the artifacts were acceptable [34]. Cone-beam CT may therefore be useful in future studies the 1-IODs.

With regard to prosthetic complications, half the present patients had fewer than 2 nylon replacements. The nylon insert was easily replaced, and the procedure time at chairside was minimal. The locator was a useful attachment for patients able to use it correctly.

However, despite appropriate retention, frequent nylon insert replacements were required for some elderly patients because of early loss of retention or deformation of the nylon insert; these patients could not use the locator correctly despite repeated practice. The locator may pose a challenge for patients with poor manual dexterity $[11,22,35]$. Another reason is the absence of a "snap-in" sound, which was reported by Kleis et al. in their study of 2-IODs [6]. The absence of an auditory cue might cause difficulties for patients using 1-IODs who need to determine if dentures are in the appropriate position. Inappropriate denture placement could result in early nylon deformation. In such cases, food debris and plaque collect in the locator. In this study, correct placement of dentures was facilitated in patients with poor dexterity by using a resin guiding wall created around the locator attachment at the chairside. After this modification, patients were able to satisfactorily manipulate their dentures by using the locator, and the number of attachments requiring replacement decreased. However, resin guiding walls are undesirable because they transmit excessive loads to the abutments and implants during mastication. A magnet attachment seems technically appropriate for such patients because it simplifies denture wear and removal. Frequent repositioning of the metal housing was required because of errors during pick-up of the locator into a denture. The intraoral procedure was technique-sensitive and more difficult to perform in patients with a flat mandibular ridge, which made dentures unstable. Complications involving the metal housing were not mentioned in a previous clinical study of 2-IODs. However, a large-scale retrospective study of implant-supported removable dental prostheses reported frequent problems with repositioning of the metal housing, especially during the first year [36].

This study has some limitations. The outcomes were obtained by using a crossover protocol of IOD with locator attachment and complete dentures with a healing abutment [23]. Ten of the 21 patients used a 1-IOD and 11 used complete dentures for the first 2 months; the groups were then crossed over to the alternative intervention for the next 2 months. At the end of the crossover period, all participants chose to use a locator attachment. The success of the nylon replacements could not be evaluated in 10 patients because of the 2-month period of complete-denture use between the initial and later IOD use. The abutment was repeatedly attached and removed during the crossover protocol, so marginal bone loss might have been greater for this method than for the conventional method. Duration of follow-up was relatively short, and longer follow-up might be needed in order to clarify long-term 1-IOD outcomes in relation to bone loss. Although there was no limitation for maxillary dentition in this study, variation in participant maxillary dentition could have resulted in inconsistent loading conditions on the lower denture. Future studies of the amount of marginal bone loss, the number of sessions needed to maintain locator attachment, and implant stability will require larger samples of patients with 1-IODs and longer follow-up.

In conclusion, cumulative implant survival and implant stability rates were high in this preliminary study of patients with 1-IODs. Values for radiographic and crestal bone loss significantly differed between the start of implant loading and 12 months postoperatively, except on the buccal and lingual sides. The need for replacement of the nylon was the most common prosthetic complication.

\section{Acknowledgments}

The authors gratefully acknowledge Drs. Anna Miyayasu and Ryo Shimada for their assistance with the prosthetic treatment and data collection.

\section{Conflict of interest}

The authors have no conflict of interest to declare.

\section{References}

1. Feine JS, Carlsson GE, Awad MA, Chehade A, Duncan WJ, Gizani S et al. (2002) The McGill consensus statement on overdentures. Mandibular two-implant overdentures as first choice standard of care for edentulous patients. Montreal, Quebec, May 24-25, 2002. Int J Oral Maxillofac Implants 17, 601-602.

2. Thomason JM, Kelly SAM, Bendkowski A, Ellis JS (2012) Two implant retained overdentures-a review of the literature supporting the McGill and York consensus statements. J Dent 40, 22-34.

3. Petersen PE, Yamamoto T (2005) Improving the oral health of older people: the approach of the WHO global oral health programme. Community Dent Oral Epidemiol 33, 81-92.

4. Bryant SR, Walton JN, Macentee MI (2015) A 5-year randomized trial to compare 1 or 2 implants for implant overdentures. J Dent Res 94, 36-43.

5. Chung K-H, Chung C-Y, Cagna DR, Cronin RJ (2004) Retention characteristics of attachment systems for implant overdentures. J Prosthodont 13, 221-226.

6. Kleis WK, Kämmerer PW, Hartmann S, Al-Nawas B, Wagner W (2010) A comparison of three different attachment systems for mandibular two-implant overdentures: one-year report. Clin Implant Dent Relat Res 12, 209-218.

7. Cristache CM, Muntianu LA, Burlibasa M, Didilescu AC (2014) Five-year clinical trial using three attachment systems for implant overdentures. Clin Oral Implants Res 25, 171178.

8. Engelhardt F, Zeman F, Behr M, Hahmel S (2016) Prosthetic complications and maintenance requirements in locator-attached implant-supported overdentures: a retrospective study. Eur J Prosthodont Restor Dent 24, 31-35.

9. ELsyad MA, Abo Hatem OE, Shawky AF, Emera RMK (2018) Effect of different degrees of mesial implant inclination on the retention and stability of two-implant mandibular over- 
dentures retained with stud attachments: an in vitro study. Int J Oral Maxillofac Implants 33, 259-268

10. ELsyad MA, Emera RM, Ashmawy TM (2019) Effect of distal implant inclination on dislodging forces of different locator attachments used for mandibular overdentures: an in vitro study. J Prosthodont 28, 666-674.

11. Alsabeeha NH, Payne AG, De Silva RK, Thomson WM (2011) Mandibular single-implant overdentures: preliminary results of a randomised- control trial on early loading with different implant diameters and attachment systems. Clin Oral Implants Res 22, 330-337.

12. Cheng T, Sun G, Huo J, He X, Wang Y, Ren Y (2012) Patient satisfaction and masticatory efficiency of single implant-retained mandibular overdentures using the stud and magnetic attachments. J Dent 40, 1018-1023.

13. Alqutaibi AY, Kaddah AF, Farouk M (2017) Randomized study on the effect of singleimplant versus two- implant retained overdentures on implant loss and muscle activity: a 12-month follow-up report. Int J Oral Maxillofac Surg 46, 789-797.

14. Krennmair G, Ulm C (2001) The symphyseal single-tooth implant for anchorage of a mandibular complete denture in geriatric patients: a clinical report. Int J Oral Maxillofac Implants 16, 98-104

15. Liddelow GJ, Henry PJ (2007) A prospective study of immediately loaded single implantretained mandibular overdentures: preliminary one-year results. J Prosthet Dent 97 , 126-137.

16. Kronstrom M, Davis B, Loney R, Gerrow J, Hollender L (2009) A prospective randomized study on the immediate loading of mandibular overdentures supported by one or two implants: a 12-month follow-up report. Int J Oral Maxillofac Implants 25, 181-188.

17. Tavakolizadeh S, Vafaee F, Khoshhal M, Ebrahimzadeh Z (2015) Comparison of margina bone loss and patient satisfaction in single and double-implant assisted mandibular overdenture by immediate loading. J Adv Prosthodont 7, 191-198.

18. Albrektsson T, Zarb GA (1993) Current interpretations of the osseointegrated response: clinical significance. Int J Prosthodont 6, 95-105.

19. Roos J, Sennerby L, Lekholm U, Jemt T, Gröndahl K, Albrektsson T (1997) A qualitative and quantitative method for evaluating implant success: a 5-year retrospective analysis of the Brånemark implant. Int J Oral Maxillofac Implants 12, 504-514.

20. Brochu J-F, Anderson JD, Zarb GA (2005) The influence of early loading on bony crest height and stability: a pilot study. Int J Prosthodont 18, 506-512.

21. Emami E, Cerutti-Kopplin D, Menassa M, Audy N, Kodama N, Durand R et al. (2016) Does immediate loading affect clinical and patient-centered outcomes of mandibular 2-unsplinted-implant overdenture? A 2-year within-case analysis. J Dent 50, 30-36.

22. Müller F, Duvernay E, Loup A, Vazquez L, Herrmann FR, Schimmel M (2013) Implantsupported mandibular overdentures in very old adults: a randomized controlled trial. J Dent Res $92,154 \mathrm{~S}-160$
23. Kanazawa M, Tanoue M, Miyayasu A, Takeshita S, Sato D, Asami M et al. (2018) The patient general satisfaction of mandibular single-implant overdentures and conventional complete dentures. Medicine (Baltimore) 97, e10721. doi:10.1097/MD.0000000000010721.

24. Cordioli G, Majzoub Z, Castagna S (1997) Mandibular overdentures anchored to single implants: a five-year prospective study. J Prosthet Dent 78, 159-165.

25. Kern M, Att W, Fritzer E, Kappel S, Luthardt RG, Mundt T et al. (2018) Survival and complications of single dental implants in the edentulous mandible following immediate or delayed loading : a randomized controlled clinical trial. J Dent Res 97, 163-170.

26. Nedir R, Bischof M, Szmukler-Moncler S, Bernard JP, Samson J (2004) Predicting osseointegration by means of implant primary stability. Clin Oral Implants Res 15, 520-528.

27. Sennerby L, Meredith N (2008) Implant stability measurements using resonance frequency analysis: biological and biomechanical aspects and clinical implications. Periodontol 2000 47, 51-66.

28. Weinlander M, Krennmair G, Piehslinger E (2009) Implant prosthodontic rehabilitation of patients with rheumatic disorders: a case series report. Int J Prosthodont 23, 22-28.

29. Krennmair S, Weinländer M, Forstner T, Krennmair G, Stimmelmayr M (2016) Factor affecting peri-implant bone resorption in four implant supported mandibular full-arch restorations: a 3-year prospective study. J Clin Periodontol 43, 92-101.

30. Misch CE (1998) The implant quality scale : a clinical assessment of the health-disease continuum. Oral Health $88,1-17$

31. Liu J, Pan S, Dong J, Mo Z, Fan Y, Feng H (2012) Influence of implant number on the biomechanical behaviour of mandibular implant-retained / supported overdentures : a three-dimensional finite element analysis. J Dent 41, 241-249.

32. Elsyad MA, Elhddad AA, Khirallah AS (2016) The effect of implant diameter on strain around implants retaining a mandibular overdenture with locator attachments: an in vitro study. Dent Mater J 35, 938-945.

33. Elsyad MA, Abid KS, Elkhalek EA (2017) Effect of buccal implant inclination on stresses around two-implant-retained overdentures with resilient stud attachments. Int J Oral Maxillofac Implants 32, 135-142

34. Elsyad MA, Khirallah AS (2016) Circumferential bone loss around splinted and nonsplinted immediately loaded implants retaining mandibular overdentures: a randomized controlled clinical trial using cone beam computed tomography. J Prosthet Dent 116, 741 748

35. Mackie A, Lyons K, Thomson WM, Payne AG (2011) Mandibular two-implant overdentures: three-year prosthodontic maintenance using the locator attachment system. Int J Prosthodont 24, 328-331.

36. Guédat C, Nagy U, Schimmel M, Müller F, Srinivasan M (2018) Clinical performance of LOCATOR $®$ attachments: a retrospective study with 1-8 years of follow-up. Clin Exp Dent Res 4, 132-145. 